\title{
VARICELLA ZOSTER VIRUS FETOPATHY - CLINICAL CASE
}

\author{
Ioana Grigore', Georgeta Diaconu ${ }^{1}$, N. Nistor ${ }^{2}$, Ioana Cernescu ${ }^{3}$, Irina Ciomaga ${ }^{2}$ \\ ${ }^{1}$ Pediatric Neurology Clinic, ,, Sf. Maria “ Children's Emergency Hospital, Iasi \\ ${ }^{2} 1^{\text {st }}$ Clinic of Pediatrics, ,, Gr. T. Popa " University of Medicine and Pharmacy, Iasi \\ ${ }^{3}$ Radiology Laboratory, ,,Sf. Maria “ Children's Emergency Hospital, Iasi
}

\begin{abstract}
Varicella zoster virus fetopathy occurs in infants after infection with varicella-zoster virus from mother during the first two trimesters of pregnancy. The authors describe the case of a female infant, aged 4 months, which came under observation for neuro developmental delay for chronological age. Neurological exam, abnomalities detected on ophthalmologic examination, maternal history of gestational varicella and increased IgG titer to varicella-zoster virus supported the diagnosis of varicella zoster virus fetopathy. Varicella zoster virus fetopathy is a condition characterized by severe skin damage, ocular, skeletal and nervous system anomalies to whom the primary prophylaxis is extremely important. In rare cases, like that presented, the clinical picture may be incomplete only neurological and ophthalmic manifestations was present.
\end{abstract}

Keywords: varicella zoster fetopathy, neurological symptoms, ocular abnormalities, primary prophylaxis

Varicella zoster fetopathy, first described by Laforet and Lynch in 1947, is a rare disease determined by the varicella-zoster virus after maternal varicella onset during the first two trimesters of pregnancy. The literature reports to an estimated 100 infants diagnosed with varicella zoster fetopathy more than $3 / 4$ of cases being reported in the last 20 years (Sauerbrei, 2003). Currently the disease incidence is estimated at $2 \%$ of newborns (Harish, 2010).

Clinical manifestations are represented by the skin lesions, neurological disorders, eye damage and skeletal abnormalities. Approximately $30 \%$ of infants which presenting a complete clinical picture dies in the first months of life (Sauerbrei, 2003). The diagnosis is supported by maternal history of varicella in the first two trimesters of pregnancy and the presence of suggestive clinical manifestation in the newborn.

\section{CLINICAL CASE}

AF, female, aged 4 months, born at term, from unrelated parents, with $\mathrm{G}=2,500 \mathrm{~g}$ and Apgar $=8$, apparently without particular historical data regarding previous ante/perinatal antecedents reach our observation for neurological assessment.

General physical examination revealed somatic slight deficit.

Neurological examination revealed neuromotor retardation (inability to align the head and thorax in prono position, lack of trunk control in prono position and lack of eye-hand-mouth coordination), fluctuating muscle tone, polikinetics osteo-tendinous reflexes at lower limb, reflexes archaic absent.

Eye examination revealed partial atrophy of the optic nerve.

The patient was investigated neuroimaging, brain CT-scan revealed fronto-parietal subarach- 
noid bilateral fluid accumulation; absence of the expansive intracranial arterial-venous malformations; ventricular system with normal morphology and uniform content of CSF (Fig. 1).

Hematologic balance was normal, biochemical balance blood revealed mild hepatic cytolysis.

TORCH syndrome serology was negative.

From the resumption anamnesis revealed that the mother presented, in the second trimester of pregnancy, chickenpox that occurred after fever, being located on the trunk and limbs. Symptoms began after contact with a child who had chickenpox. The mother was diagnosed with gestational varicella and received only treatment with anti-pyretic.

Immunological examination revealed elevated IgG varicella-zoster virus $(730 \mathrm{mUI} / \mathrm{ml})$.

Anamnesis, abnormalities recorded to neurological and to the eye examination associated with the presence of elevated serum IgG to varicellazoster virus led to the diagnosis of varicella zoster virus fetopathy. The indications at hospital discharge were global stimulation, proper prevention of rachitis, age-appropriate diet and for 1 month hepatoprotective treatment.

At the age of 7 months when she presented for diagnosis of development neurological exam revealed severe neuromotor retardation, fluctuating muscle tone, ROT polikinetics and psychomotor agitation.

\section{DISCUSSIONS}

Chickenpox contacted during pregnancy can cause serious maternal and fetal complications

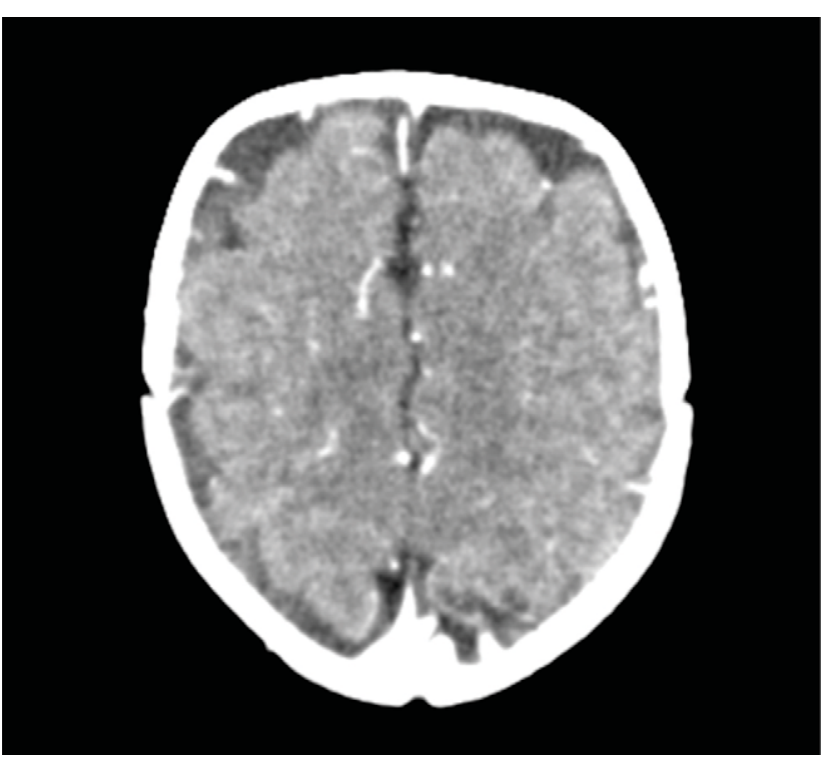

FIGURE 1. Brain CT-scan revealed fronto-parietal subarachnoid bilateral fluid accumulation whose severity depends on the time of onset of disease at the pregnant woman. If occurs in the first 20 weeks of gestation fetal risk of varicella zoster virus fetopathy is $2 \%$ with a mortality of $30 \%$ (Sauerbrei, 2001). If chickenpox occurs in the third trimester of pregnancy may increase the risk of neonatal varicella up to $50 \%$. Currently there is no consensus regarding antiviral treatment of chickenpox during pregnancy and its effectiveness in preventing or decreasing the severity of varicella zoster virus fetopathy. Harish (2010) suggests the use of antiviral drugs to pregnant women diagnosed with chickenpox only in cases where the benefit to the mother outweighs the risk to the fetus because the treatment with acyclovir is classified as category $\mathrm{C}$ by Agency of Food and Drug Administration concerning the teratogenicity risk. Also, American Academy of Pediatrics recommends treatment with acyclovir in pregnant women who contacted chickenpox only in cases complicated with pneumonia or encephalitis, when antiviral medication administration will be done intravenously. In the case presented mother was diagnosed with chickenpox in the second trimester of pregnancy, received only anti-pyretic treatment.

Since both varicella zoster fetopathy and neonatal varicella are serious illness and infants affected are poor prognosis for neuropshychomotric developing, prophylaxis of gestational varicella is very important. Many authors indicate immunization with live-attenuated varicella vaccine at nonpregnant women of child-bearing age without a history of varicella, considering that this ensures complete protection against severe forms of chickenpox. For at least 4 months pregnancy should be avoided because live-attenuated varicella vaccine is contraindicated in pregnant woman (Sauerbrei, 2001). Also, in cases where a pregnant woman has come into contact with people with chickenpox immunization with inactivated virus must be done within 72-96 hours of exposure, as recommended by the Robert Koch Institute in 2000. Although passive immunization of pregnant women prevents the disease at mother and their complications and may theoretically reduce the risk of fetal infection, there is no evidence that prevents varicella zoster fetopathy.

The literature data contend that injuries during fetal varicella-zoster virus are stationary because there is no postnatal viral replication at that moment (Kohli, 2006). Varicella-zoster virus, as the causative agent of varicella zoster virus fetopathy, can revealed rare in children. From the literature review, Sauerbrei (2003) noted that in only $60 \%$ of cases have been demonstrated intrauterine infection with this virus. 
Clinical varicella zoster virus fetopathy is characterized by signs and symptoms due to damage skin, nervous system and osteoarticular system. Sauerbrei (2003) observed that skin lesions are present in $76 \%$ of cases, neurological defects in $60 \%$, eye lesions in $51 \%$ of cases and skeletal abnormalities in $49 \%$ of patients. Also, Andreas (2000) noted that skin lesions are the most common, being recorded at 3 of 4 children and eye disease and nervous system that occurs in about $1 / 2$ and $2 / 3$ of patients. Impaired skin is the zigzag scar that generally complies distribution of dermatomes (Harish, 2010). Ocular involvement may cause cataracts, chorioretinitis, corneal opacities, microphthalmia and optic atrophy (Myers, 2007). In varicella zoster fetopathy neurological manifestations may be caused by cerebral atrophy or, in some cases, lesions occur in the sympathetic fibers in the cervical and/or lumbosacral cord what causes Horner's syndrome and sphincter disorders (Harish, 2010). In rare cases, were described only cerebral and ocular manifestations (Sauerbrei, 2003). In the described case the child has not had skin lesions, but showed signs of ocular affectation represented by partial atrophy of the optic nerve and neurological disorders manifested by neuropsychomotor retardation.

The diagnosis of varicella zoster virus fetopathy is supported by the history of gestational varicella associated with clinical signs observed in newborns
(Myers, 2007). In the presented case the diagnosis of varicella zoster virus fetopathy was made at 4 months of age when the child showed clinical signs of neurological and ocular affectation and medical history revealed the presence of maternal varicella in the second trimester of pregnancy. Positive serology for varicella-zoster virus supported the diagnosis.

\section{CONCLUSIONS}

Varicella zoster virus fetopathy is a condition characterized by severe skin lesions, ocular and neurological signs. In rare cases, like that presented, clinical manifestations may include only ocular and neurological abnormalities. The diagnosis is mainly based on positive history of gestational varicella, on the clinical signs and positive serology for varicella-zoster virus. Because varicella zoster virus fetopathy is a severe disease, the primary prophylaxis is extremely important. So, at the routine control of a young woman who wants to become a mother should be performed serology for varicellazoster virus, and if it is negative immunization should be offered. In cases where a pregnant woman without a history of chickenpox has had a significant exposure to this illness she should be immunized, after she is informed that this form of prevention may not reduce the risk of infection to the fetus.

\section{REFERENCES}

1. Sauerbrei A., Pawlak J., Luger C., Wutzler P. Intracerebral varicella-zoster virus reactivation in congenital varicella syndrome. Dev Med Chil Neurol. 2003; 45(12):837-40.

2. Harish R., Jamwal A., Dang K. Congenital varicella syndrome/ Varicella Zoster Virus Fetopathy. Indian Journal of Pediatrics. 2010; 77:92-93.

3. Sauerbrei A., Wutzler P. Neonatal varicella. Journal of Perinatology. 2001; 21:545-549.

4. Robert Koch - Institut: Ratgeber Infektionskrankheiten. 19 Folge: Varizellen, Herpes zoster. Epidemiol Bull. 2000; 46:365-8.

5. Kohli D., Rana N. Congenital varicella syndrome: preseting with eye complications. Indian Pediatrics. 2006; 43:653-654.

6. Andreas S., Peter W. The congenital varicella syndrome. J Perinatol. 2000; 20:548-554.

7. Myers M.G., Seward J.F., LaRussa P.S. Varicella zoster virus in: Berman R.E., Kleigman R.M., Jenson H.B., Stanton B.F. ed. Nelson Textbook of Pediatrics. $18^{\text {th }}$ ed. Philadelphia: Saunders 2007; 1366-1372. 